\title{
Angst bei Augustin
}

Volker Henning Drecoll

Kaum ein theologischer Denker hat sich so intensiv mit Emotionen beschäftigt wie Augustin. Die innere Zerrissenheit des Menschen, aber auch die Angst vor dem Tod und vor Gebrechen, aber auch vor dem eigenen Scheitern sind für ihn zentrale Gegenstände theologischer Reflexion. Zugleich ist für ihn Glaube nicht nur ein Für-Wahr-Halten oder eine Lebens- und Verhaltensweise, sondern vor allem affektiv geprägt. Dies beschreibt Augustin als Liebe und als Freude. Er findet hierin den Schlüssel, um einerseits die Passivität des Menschen gegenüber Gottes Gnade, andererseits seine innere, voluntative Beteiligung auszudrücken. ${ }^{1}$ Gefühle kann man nicht machen, und man hat sie auch nur sehr begrenzt in seiner Hand. Diese Einsicht führt Augustin nicht nur zu einer Modifikation des Apathieideals, sondern auch zu einer Beschreibung des Glaubens als Überwindung von Angst. Angst und Furcht liegen dabei im Lateinischen eng beieinander, ohne dass die im Deutschen geläufige Differenz von Angst (eher allgemein und diffus) und Furcht (eher auf ein konkretes Ereignis bezogen) in den lateinischen Substantiven metus und timor so wiederfindbar wäre, wie gerade die transitiven Verben metuere und timere zeigen.

\section{Angstlosigkeit als höchstes Ziel?}

Den grundlegenden Zusammenhang von Liebe und Angst entwickelt Augustin bereits in der Frühschrift De diuersis quaestionibus 83 (geschrieben zwischen 388 und 391) 2 $^{2}$ Augustin geht davon aus, dass Angst daraus besteht, entweder zu befürchten, dass man etwas verliert, was man liebt und schon hat, oder zu befürchten, dass man etwas verliert, was man liebt, aber noch nicht hat, sondern hofft zu haben. ${ }^{3}$ Angst hängt also grundlegend mit dem zusammen, was man liebt - und haben oder behalten möchte. Augustin fragt sich,

\footnotetext{
1 Vgl. Isabelle Bochet, Saint Augustin et le désir de Dieu, Paris 1982, 322-342.

2 Eine lateinisch-deutsche Ausgabe hat vorgelegt: Carl Johann Perl, Aurelius Augustinus, Dreiundachtzig verschiedene Fragen/De diversis quaestionibus octoginta tribus, Deutsche Augustinus Ausgabe [Nr. 6], Paderborn 1972.

3 Augustin, De diversis quaestionibus LXXXIII 33 (CCSL 44A, 47,2-4 Mutzenbecher).
} 
wie Angstlosigkeit aussehen könnte. Für den ersteren Fall, dass sich die Angst auf das richtet, was man liebt und hat, ist klar, dass man Abwesenheit von Angst nicht so haben kann, dass man sie zugleich ängstlich bewahrt. Normalerweise bewahrt man ja ängstlich, was man hat und liebt, aber für die Angstlosigkeit ist das nicht denkbar. ${ }^{4}$ In ähnlicher Weise kann man auch nicht befürchten, dass man die Angstlosigkeit nicht erreicht, denn dadurch würde man ja gerade eine angstbesetzte Haltung einnehmen, also gerade verhindern, dass das, was man liebt und haben möchte, eintreten kann. ${ }^{5}$ Angstlosigkeit als Ziel des eigenen Strebens, als das, was man liebt und hat oder erhofft, ist also nur möglich, wenn sie bereits da ist. Weder kann man Angstlosigkeit ängstlich bewahren noch ängstlich erhoffen.

Angstslosigkeit, so Augustin, wirkt sich auch auf andere Gefühle aus. Einer stoischen Affektenlehre folgend, die er aus Ciceros Tusculanae Disputationes entnehmen konnte, ${ }^{6}$ nennt Augustin insbesondere drei negative Gefühle: Begierde, Niedergeschlagenheit und leere Fröhlichkeit. Angstlosigkeit bringt auch diese Gefühle zum Erliegen. Denn Begierde ist die Liebe zu vergänglichen Dingen, so dass sie von der Angst, sie zu verlieren, geprägt ist - und ohne die entsprechende Angst gar nicht zu denken ist. Die Niedergeschlagenheit (oder Traurigkeit) realisiert den entsprechenden Verlust nur schon, den die Angst für die Zukunft befürchtet. Und die leere Fröhlichkeit zeichnet sich ja dadurch aus, dass sie sich über Dinge freut, die man eben auch verlieren kann. Angstlosigkeit wirkt sich also umfassend aus, sie erfasst auch andere negative Gefühle und drängt sie zurück. ${ }^{7}$ Um so wichtiger ist die Frage, wie man Angstlosigkeit erreicht und ob sie das eigentliche Ziel menschlichen Strebens sein soll.

Dem geht Augustin nach, indem er die Frage stellt, ob die Abwesenheit von Angst als solche erstrebenswert ist - oder ob man nicht doch noch weitere Zusatzbedingungen formulieren muss. Das ist sehr wohl der Fall, wie Augustin an zwei Beispielen zeigt, bei denen deutlich ist, dass keine Angst vorhanden ist, die jedoch keineswegs erstrebenswert sind. Das ist zum einen das Beispiel des Wagemutigen oder dreist Handelnden, der sich voreilig in seine Handlungen stürzt, dem es also an Vorsicht und Umsicht fehlt, zum anderen das

\footnotetext{
4 Augustin, De diversis quaestionibus LXXXIII 33 (CCSL 44A, 47,5-8 Mutzenbecher).

${ }^{5}$ Augustin, De diversis quaestionibus LXXXIII 33 (CCSL 44A, 47,8-11 Mutzenbecher).

${ }^{6}$ Cicero, Tusculanae disputationes 4,6,11-14 (Bibliotheca Teubneriana M. Tullius Cicero Fasc. 44, 366,21-368,8 Pohlenz).

7 Augustin, De diversis quaestionibus LXXXIII 33 (CCSL 44A, 48,25-36 Mutzenbecher).
} 
Beispiel des toten Kadavers, der sicherlich keine Angst hat, weil er ja überhaupt gar keine Sinneswahrnehmungen hat. ${ }^{8}$ Beide Beispiele zeigen:Vermeidung von Angst als solche kann nicht höchstes Ziel des menschlichen Bemühens sein, vielmehr muss man zwei Zusatzbedingungen formulieren, nämlich a) dass es sich um die Gestaltung des Lebens handelt (und nicht um die Beendigung desselben) und b) dass sich dieses Leben mit Einsicht undVerstehen verbindet, selbst durchdacht ist. Ziel menschlichen Strebens ist es also: ohne Furcht, aber mit Einsicht zu leben (sine metu cum intellectu uiuere). ${ }^{9}$

Was hier als Ziel menschlichen Strebens umschrieben wird, beschreibt Augustin als Liebe. Ohne Angst, aber mit Einsicht zu leben ist Gegenstand des Liebens, es ist zu lieben (amandum est). Augustin fragt sich, ob dies bedeutet, dass zu dieser Liebe auch die Liebe zu dieser Liebe gehört, ob diese Liebe also eine Selbstbezüglichkeit beinhalten muss, die dann also zu dem Ziel angstlos, aber mit Verstand zu leben hinzuzunehmen wäre. ${ }^{10}$ Das wäre ja dann zu bejahen, wenn ohne eine solche Liebe das Ziel (also: sine metu cum intellectu vivere) nicht geliebt werden könnte. Allerdings setzt das dann voraus, dass die Liebe nicht verzweckt wird, denn Liebe ist eigentlich nur dann gegeben, wenn etwas um seiner selbst willen geliebt wird. Daher scheidet einerseits eine Instrumentalisierung der Liebe aus, denn eine solche würde den Charakter der Liebe beeinträchtigen, sie ist dann eben ein planvolles Streben, aber nicht wirklich Liebe. Andererseits würde aber auch eine bloße Selbstbezüglichkeit der Liebe nicht ausreichen, denn bei ihr wäre nicht gesichert, dass der Gegenstand der Liebe immer gegeben wäre. ${ }^{11}$ Das führt zu dem Ergebnis: Angstlosigkeit ist nur möglich, wenn a) ein Leben geschieht, das b) mit Einsicht geführt wird und sich c) liebend auf etwas bezieht, das nicht verloren werden kann. Dieses ist nur möglich, wenn der Bezugspunkt des menschlichen Strebens nicht im Menschen selbst verankert ist, sondern außerhalb des Menschen gegeben ist.

\footnotetext{
8 Augustin, De diversis quaestionibus LXXXIII 34 (CCSL 44A, 49,2-12 Mutzenbecher).

9 Augustin, De diversis quaestionibus LXXXIII 35,1 (CCSL 44A, 50,2-6 Mutzenbecher).

${ }^{10}$ Diese Überlegung ist ein Korrelat zu der Überlegung, ob man auch vor der Angst selbst Angst haben kann - was Augustin zufolge nicht der Fall ist, weil sich Angst immer auf die Zukunft bezieht, also man vielleicht vor einem zukünftigen Angstzustand Angst haben kann, aber in dem Moment der Angst die Angst als solche da ist, ohne selbstbezüglich Gegenstand der Angst zu sein, vgl.Augustin, De diversis quaestionibus LXXXIII 33 (CCSL 44A, 47,12-22 Mutzenbecher).

${ }_{11}$ Augustin, De diversis quaestionibus LXXXIII 35,1 (CCSL 44A, 50,6-19 Mutzenbecher).
} 
Damit legt Augustin eine ontologische Grundstruktur frei, die auf eine grundlegende Abhängigkeit des Menschen verweist. Das Ziel des menschlichen Strebens kann letztlich nicht im Menschen selbst gegeben sein, sondern liegt in etwas Ewigem außerhalb des Menschen, auf das sich der Mensch in richtiger Weise beziehen muss. ${ }^{12}$ Hierfür macht Augustin zwei Bedingungen geltend:

a) Der liebende Bezug auf das Ewige beschränkt sich nicht auf die Erkenntnis durch die höchste Instanz im Menschen, die mens oder ratio, mit der der Mensch ewige Sachverhalte erkennen kann. Denn der Verstand hat nur dann die Möglichkeit, das Ewige zu betrachten, wenn die anderen Seelenteile mit ihm übereinstimmen und ihm so in einer gewissen Ruhe und Friedlichkeit den Raum für die Betrachtung geben; Liebe ist also umfassender als das Erkennen:Während nur derVerstand das Ewige bedenken kann, muss der liebende Bezug auf das Ewige die gesamte Seele umfassen und hier Ruhe und Frieden (pax) erreichen. ${ }^{13}$ Richtiges Erkennen ist nur mit Liebe möglich, was eben nicht nur den Verstand betrifft, sondern den Menschen insgesamt einbezieht, also gerade auch eine affektive Seite umfasst.

b) Das, was geliebt wird, muss selbst den Liebenden affizieren. Im Falle eines Bezugs auf etwas Ewiges muss dieses Affizieren selbst in Ewigkeit geschehen. Ein seliges Leben ist also strikt nur als ewiges Leben denkbar. Damit ist aber deutlich, dass derjenige, der den Verstand ewig affiziert, selbst ewig sein und ewig handeln muss, also Gott ist. ${ }^{14}$ Damit wird deutlich, dass Gott nicht einfach nur Gegenstand der Erkenntnis und Liebe ist, sondern seinerseits aktiv auf diese beiden miteinander verbundenen Prozesse einwirken muss, damit das Ziel des menschlichen Strebens erreicht werden kann. Das bedeutet auch: Der Mensch kann das Ziel nicht von sich aus erreichen, sondern nur dann, wenn Gott auf ihn entsprechend einwirkt. Zugleich wird deutlich, dass dieses Handeln Gottes erst in einem ewigen Leben zu seinem Ziel kommt.

\footnotetext{
${ }^{12}$ Dieses externe Ziel kann nicht durch den Bezug auf äußerliche, etwa körperliche Gegenstände, aber auch nicht durch bloßes Wissen (etwa eine Zahlenartistik oder andere Kenntnisse zum Geld- oder Ansehenserwerb) erreicht werden, denn ein vollkommenes Haben ist nicht ohne Liebe möglich, sonst würde es sich nicht um ein höchstes Ziel handeln, sondern nur um einen Mittel zum Zweck, vgl. Augustin, De diversis quaestionibus LXXXIII 35,1 (CCSL 44A, 51,21-39 Mutzenbecher).

${ }_{13}$ Augustin, De diversis quaestionibus LXXXIII 35,2 (CCSL 44A, 52,50-56 Mutzenbecher).

${ }^{14}$ Augustin, De diversis quaestionibus LXXXIII 35,2 (CCSL 44A, 52,56-60 Mutzenbecher).
} 
Die Reflexion, ob Angstlosigkeit als solche anzustreben, d.h. zu lieben ist, führt Augustin zu einer grundlegenden Reflexion über den Charakter und den Gegenstand einer Liebe, die ohne Verlust geschieht. Etwas vereinfacht könnte man sagen: Angst ist nur durch Liebe ablösbar, und zwar eine Liebe, die sich auf etwas bezieht, das nicht verloren werden kann, also Gott. Diese Liebe ist nur möglich, wenn Gott selbst nicht nur den Verstand, sondern die Seele des Menschen insgesamt berührt. Gnade ist daher nicht nur Erkenntnis, sondern notwendigerweise affektiv. Und sie kann sich nicht auf das diesseitige, vergängliche Leben beschränken, sondern findet erst in einem eschatologischen Zustand des ewigen Lebens seine Vollendung. Damit ist eine Anthropologie entworfen, die den Menschen von seinem Gottesbezug her versteht und diesen Gottesbezug als von Gott verursachte Bewegung auf Gott hin beschreibt. Augustins Gnadenlehre lässt sich als Versuch verstehen, diese Grundbestimmungen konsequent zu durchdenken.

\section{Die Liebe löst die Angst ab}

Dementsprechend kann Augustin im Zusammenhang des pelagianischen Streits ${ }^{15}$ seine Gnadenlehre in der Schrift De spiritu et littera (Vom Geist und Buchstaben) ${ }^{16}$ insbesondere auch durch die Abfolge von Angst und Liebe beschreiben. Exegetische Grundlage ist ihm dafür insbesondere Röm 8,15: »Ihr habt nicht empfangen den Geist der Knechtschaft, der zurück in die Angst führt, sondern den Geist der Adoption zu Söhnen, in dem wir rufen:Abbas, Vater«. Dies wendet Augustin auf die Unterscheidung zwischen Altem und Neuen Bund an. Alter und Neuer Bund lassen sich nicht schematisch auf Altes und Neues Testament verteilen, gemeint ist vielmehr eine unterschiedliche Wirkweise.

Dabei geht Augustin in drei Schritten vor. Zuerst stellt er die Gesetzesbefolgung, die aus Angst vor Strafe geschieht, derjenigen, die aus Liebe zur Gerechtigkeit geschieht, gegenüber: Gerade mit

\footnotetext{
15 Vgl.Volker Henning Drecoll, Art. Gratia, in: Cornelius Petrus Mayer (Hg.), Augustinuslexikon, Bd. 3, Basel 2004-2010, 182-242, hier 202-209; Art. Pelagius, Pelagiani, in: Cornelius Petrus Mayer/Robert Dodaro/Christof Müller (Hg.), Augustinuslexikon, Bd. 4, Basel/Berlin 2012-2018, 624-666, hier 631-632.

${ }^{16}$ Es existieren zwei lateinisch-deutsche Ausgaben, einerseits Anselm Förster, Geist und Buchstabe. De spiritu et littera liber unus, Deutsche Augustinus Ausgabe, Paderborn 1968; andererseits Sebastian Kopp, Der Geist und der Buchstabe, in: Aurelius Augustinus. Schriften gegen die Pelagianer, Bd. 1: Lateinisch-deutsch (Sankt Augustinus. Der Lehrer der Gnade, Bd. 1), Würzburg 1971, 303-435. 653-682.
} 
einer Gesetzesbefolgung aus Angst erfüllen die Menschen das Gesetz eigentlich nicht, weil Gott weiß, dass sie eigentlich, wenn es ungestraft geschehen könnte, anders handeln würden. ${ }^{17}$ Das kann erst durch den hinzutretenden Geist, der hilft und heilt, verändert werden, weil dann eine »Beschneidung des Herzens" (Röm 2,29) erfolgt, also eine innere Ausrichtung des Menschen, die frei ist von jeglichem Begehren nach Verbotenem. ${ }^{18}$ Zweitens stellt Augustin fest, dass durch diesen Wechsel, wenn die Liebe die Angst ablöst (cum timori successerit $\operatorname{caritas}^{19}$ ), nicht das Gesetz als solches aufgelöst wird (auch wenn die irdisch-fleischlichen Verheißungen durch geistige ersetzt und die Ritualgesetze nicht mehr bindend sind), sondern dass aus dem Gesetz der Werke ein Gesetz des Glaubens wird. Mit dieser aus Röm 3,27 stammenden Unterscheidung sei, so Augustin, eine unterschiedliche Wirkweise gemeint: Quod operum lex minando imperat, hoc fidei lex credendo impetrat (Was das Gesetz der Werke durch Drohungen befiehlt, das erbittet das Gesetz des Glaubens durch den Glauben). ${ }^{20}$ Das Drohen führt zu einer sklavischen Einhaltung des Gesetzes, und eben nicht einer freien, freiwilligen Beachtung der Gebote, damit aber wird das Gesetz eben eigentlich gar nicht erfüllt (seruiliter fit, non liberaliter et ideo nec fit). ${ }^{21}$ Das ändert sich nur, wenn der Glaube, der durch Liebe tätig ist (Gal 5,6), beginnt, innerlich mit dem Gesetz übereinzustimmen (Röm 7,22). An die Stelle der Bemühung um den (toten) Buchstaben tritt dann der lebendigmachende und mit Freiheit verbundene Geist, auch wenn der Glaubende in seinem Leben noch nach wie vor belastet ist von innerer Zerrissenheit (dem anderen Gesetz, das noch in ihm wirkt, aus Röm 7,23). Augustin nimmt hier eine von Tag zu Tag zunehmende Erneuerung im Inneren des Menschen an, die endzeitlich erst zu ihrem Ziel finden wird, und verbindet dies mit dem corpus mortis (dem Leib, der dem Tod unterworfen bzw. ausgesetzt ist). ${ }^{22}$ In der Schrift De peccatorum meritis et remissione hatte Augustin insbesondere auch die Angst vor dem Tode als Gegenstand des Glaubenskampfes bezeichnet, in dem sich die Gläubigen bewäh-

\footnotetext{
${ }^{17}$ Augustin, De spiritu et littera 13 (CSEL 60, 165,18-25 Urba/Zycha).

18 Augustin, De spiritu et littera 13 (CSEL 60, 165,25-166,3 Urba/Zycha).Vgl. Colten Cheuk-Yin Yam, Trinity and Grace in Augustine. An analysis of De trinitate 8-10 in Light of De spiritu et littera, Bd. 10, Paderborn 2019, 447.

${ }_{19}$ Augustin, De spiritu et littera 31 (CSEL 60, 184,15 Urba/Zycha).

${ }^{20}$ Augustin, De spiritu et littera 22 (CSEL 60, 175,13-14 Urba/Zycha).

${ }^{21}$ Augustin, De spiritu et littera 26 (CSEL 60, 180,22-24 Urba/Zycha).

${ }_{22}$ Augustin, De spiritu et littera 26 (CSEL 60, 180,25-181,4 Urba/Zycha).Vgl.Yam, Trinity and Grace, 477-480.
} 
ren. ${ }^{23}$ Damit ist deutlich, dass auch der Glaubende immer noch angefochten und der Sünde unterworfen bleibt, sei es, dass er wirklich sündhaft handelt, weil das Begehren sich über die "Riegel der Angst" (repagula timoris) hinwegsetzt, sei es, dass er es nur will, aber nicht umsetzt, weil sich die Angst vor Strafe (timor poenae) doch über die verlockende Begierde hinweggesetzt hat. ${ }^{24}$ Sündlosigkeit ist damit im diesseitigen Leben nicht möglich, erst in einer eschatologischen Vollendung wird daher auch die Angst wirklich vollständig durch Liebe ersetzt sein.

In einem dritten Schritt räumt Augustin ein, dass natürlich auch die, die aus Angst das Gesetz beachten und so ihre eigene Gerechtigkeit aufrichten wollen (nach Röm 10,3), eine gewisse Gerechtigkeit haben. Sie erreichen natürlich nicht die Gerechtigkeit Gottes, denn diese kann nur die Liebe, nicht die Angst erreichen. Die Liebe will nur das, was erlaubt ist und angemessen ist, während die Angst dazu führt, dass jemand sich zu Handlungen zwingt, während er eigentlich etwas anderes im Sinn hat, das er lieber tun würde, wenn es nicht verboten wäre. ${ }^{25}$ Auch die, in denen Angst da ist, haben also einen gewissen Glauben, denn sonst würden sie sich ja um das Gesetz gar nicht scheren. Doch ist dies eben die sklavische Haltung, die Paulus in Röm 8,15 gemeint hat, eine sklavenhafte Angst (timor seruilis), mit der die Strafe gefürchtet wird und der die Liebe zur Gerechtigkeit gegenübergestellt wird. ${ }^{26}$ Beinhaltet also einerseits die Angst, wenn sie sich auf die vom Gesetz angedrohte Strafe richtet, schon ein gewisses $\mathrm{Ma} \beta$ an Glauben, so ist dann umgekehrt auch in dem Glauben weiterhin das Dienen enthalten, und zwar werden aus den Sklaven Kinder, die freiwillig dem Vater dienen. Besonderes Kennzeichen dieses neuen Dienens ist das Gebet, das Paulus schon in Röm 8,15 genannt hatte. Die zu Kindern Adoptierten rufen Abba,Vater, und zeigen damit, von wem sie die Kraft und Fähigkeit, den Willen und die Möglichkeit, dem Willen entsprechend zu leben, erwarten, nicht von sich aus, sondern eben vom Vater. ${ }^{27}$ Augustin führt dazu das Beispiel der Enthaltsamkeit ein:Wenn das Gesetz der Sünde aufflammt und so bei sich bietender Gelegenheit die Sünde

\footnotetext{
${ }^{23}$ Augustin, De peccatorum meritis et remissione 2,51; 2,54 (CSEL 60, 122,11, 124,17-18 Urba/Zycha).Vgl. hierzu insbesondere Robert Dodaro, "Christus Iustus" and Fear of Death in Augustine's Dispute with Pelagius, in: Signum Pietatis. Festgabe für Cornelius Petrus Mayer, Würzburg 1989, 341-361.

${ }^{24}$ Augustin, De spiritu et littera 34 (CSEL 60, 187,12-16 Urba/Zycha).

${ }^{25}$ Augustin, De spiritu et littera 56 (CSEL 60, 213,1-6 Urba/Zycha).

${ }^{26}$ Augustin, De spiritu et littera 56 (CSEL 60, 213,6-13 Urba/Zycha).Vgl.Yam, Trinity and Grace, 552.

27 Augustin, De spiritu et littera 56 (CSEL 60, 213,13-27 Urba/Zycha).
} 
das Begehren befeuert, von wem ist dann die Enthaltsamkeit zu erbitten? Diese kann letztlich nur Gott geben. ${ }^{28}$ Dieses Beispiel soll zeigen, dass nicht die Angst vor Strafe oder das Bemühen, von sich selbst aus ein gerechtes Handeln erreichen zu können, wirklich zu einer Gerechtigkeit führen, die aus innerer Motivation geschieht und somit auch das ganze Wollen des Menschen umfasst. Das ist vielmehr nur möglich, wenn der Glaube durch Liebe (nicht durch Angst) tätig wird, diese Liebe aber wiederum entsteht nicht von allein, sondern nur als Gabe Gottes durch den Heiligen Geist. Diese von Gott geschenkte Liebe ist nicht einfach die Liebe Gottes zu den Menschen, sondern affiziert den inneren Menschen und löst in ihm Liebe zu Gott aus - ebenso wie die Gerechtigkeit Gottes die ist, mit der er uns zu Gerechten macht, das Heil Gottes uns heil macht, die fides Christi (die Treue Christi zu bzw. der Glaube Christi an uns) uns zu Gläubigen macht. ${ }^{29}$

\section{Die liebevolle Angst um andere}

In seinem umfangreichen Werk über die Gottesstadt ${ }^{30}$ geht Augustin im Zusammenhang der Auslegung der Paradiesesgeschichte auch auf die Emotionen ein, die Adam und Eva vor dem Fall gehabt haben. Er setzt dabei voraus, dass die grundlegenden Affekte des Menschen sich als jeweilige Färbung einer Willensrichtung (voluntas) verstehen lassen: Begierde und Fröhlichkeit sind Willensregungen, die mit Zustimmung verbunden sind. Die Begierde bezieht sich dabei auf etwas, was noch angestrebt wird, die Fröhlichkeit auf das, was bereits besessen und genossen wird. Demgegenüber sind Angst und Traurigkeit Willensregungen, die mit Abneigung (dissensio) verbunden

\footnotetext{
${ }^{28}$ Augustin, De spiritu et littera 56 (CSEL 60, 213,27-214,6 Urba/Zycha).

${ }^{29}$ Augustin, De spiritu et littera 56 (CSEL 60, 215,3-15 Urba/Zycha).

${ }^{30}$ Wegen des besonders von Johannes von Oort herausgearbeiteten Bezugs der ciuitas dei auf Jerusalem (und die biblische Zionstheologie) ist der im Deutschen geläufige Titel Gottesstaat durch Gottesstadt abzulösen (in anderen europäischen Sprachen ist dies ohnehin üblich: City of God, Cité de Dieu, Città di Dio etc.); die deutsche Übersetzung von Wilhelm Thimme, Vom Gottesstaate (De civitate dei), 2 Bände, eingeleitet von Carl Andresen, München ${ }^{2} 1985$, ist nach wie vor unersetzt. Vgl. zum Konzept von ciuitas die kurze Zusammenfassung von Johannes van Oort, Civitas dei-civitas terrena. The Concept of the Two Antithetical Cities and Its Sources, in: Augustinus. De civitate dei, hg. v. Christoph Horn, Berlin 1997, 157-169. Zur Datierung von Buch 14 auf 419 und den Zusammenhang mit der Juliandebatte vgl. Volker Henning Drecoll, Kommentar zu ep. 184A, in: Revue d'Étues Augustiniennes et Patristiques 62 (2016), 67-93, hier 86-87.
} 
sind: Hier bezieht sich Angst auf das, was noch nicht eingetreten ist, Traurigkeit auf das, was bereits eingetreten ist. Angst wird demnach eingeordnet in vier grundlegende Willensrichtungen, und als Abneigung, als Befürchtung beschrieben. ${ }^{31}$ Diese Zuordnung der vier Grundaffekte, die Augustin schon in seiner Frühschrift De diversis quaestionibus aus Cicero's Auseinandersetzung mit der stoischen Affektenlehre übernommen hatte, zur einen voluntas dient Augustin dazu, Affekte und ihre moralische Qualität zu unterscheiden. Denn inhaltlich werden diese Affekte von dem geprägt, worauf sich die voluntas richtet (sei es in Zustimmung oder Abneigung). Derjenige, der gottgemäß lebt, liebt das Gute, d.h. bei ihm richten sich Zustimmung und Abneigung nach der Liebe zu Gott und dem Guten. Bei dem Menschen, der nicht gottgemäß lebt, sondern nach eigenen Maßstäben (secundum hominem), ist das genau anders. ${ }^{32}$ Das Vorhandensein von Affekten selbst ist also wertneutral. Ein guter Wille führt zu einer guten Liebe, ein verkehrter Wille zu einer verkehrten Liebe. Die vier Affekte können demnach jeweils gut oder schlecht sein, je nachdem, wie die innere voluntas, die ihnen zugrundeliegt, geartet ist. ${ }^{33}$

Augustin fügt dem einen Schriftbeweis hinzu, mit dem er zeigen möchte, dass die Affekte auch jeweils positiv konnotiert werden können. Für die Begierde (cupiditas) führt er dazu den Begriff der concupiscentia (Begehrlichkeit) ein und illustriert an mehreren Bibelstellen, dass biblisch auch die Begehrlichkeit positiv gemeint sein kann (obwohl im alltäglichen Sprachgebrauch die Begierde, wenn sie ohne weitere Erläuterung, etwa ein Genitivattribut, auftaucht, in der Regel sich auf einen negativ zu bewertenden Affekt bezieht). Die Freude ist ohne Weiteres als positives Gefühl biblisch belegbar. Für die Angst verweist Augustin auf verschiedene Bibelstellen, nämlich Phil 2,12, Röm 11,20 und 2. Kor 11.3. Phil 2,12 (cum timore et tremore) verweist auf die Ehrfurcht und den Ernst, mit dem die Gläubigen sich um ihr Heil sorgen. ${ }^{34}$ Röm 11,20 verweist auf den Gegensatz einer äußerlichen Weisheit und der ehrfürchtigen $\mathrm{Zu}-$ stimmung zu Gottes Heilsplan. 2. Kor 11,3 kennzeichnet schließlich die anteilnehmende und fürsorgliche Angst des Apostels Paulus, dass womöglich auch die Korinther wieder von ihrer Reinheit (castitas)

\footnotetext{
${ }^{31}$ Augustin, De ciuitate dei 14,6 (CCSL 48, 421,5-13 Dombart/Kalb).

${ }_{32}$ Augustin, De ciuitate dei 14,6 (CCSL 48, 421,13-21 Dombart/Kalb).

${ }_{33}$ Augustin, De ciuitate dei 14,7 (CCSL 48, 422,40-45 Dombart/Kalb).Vgl. John C. Cavadini, Feeling Right. Augustine on the Passions and Sexual Desire, in:Augustinian Studies 36 (2005), 195-217, hier 201.

${ }^{34}$ Augustin, De ciuitate dei 14,7 (CCSL 48, 422,45-423,58 Dombart/Kalb).
} 
abgebracht werden - so wie Eva von der Schlange verführt wurde. Damit ist nicht nur der Bezug zur Paradiesesgeschichte hergestellt, sondern auch besonders verdeutlicht, dass neben der positiven Deutung der Angst als Ehrfurcht Gott gegenüber auch die anteilnehmende Angst um die Mitchristen als positive Form von Angst $\mathrm{zu}$ deuten ist. ${ }^{35}$

Für den vierten Affekt, die Traurigkeit, ist die Lage komplizierter. Augustin rekurriert hier zunächst auf die Vorstellung von den geordneten guten Affekten, die auch die stoische Affektenlehre kannte. Wiederum greift Augustin hierfür auf Cicero zurück. Die stoische Affektenlehre hatte die vier negativen Affekte, also Begierde, Fröh-

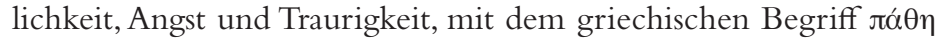
(pathē. lat. perturbationes) bezeichnet, was besonders das Erleiden dieser Affekte bezeichnet. Davon hatte sie drei gute Affektionen un-

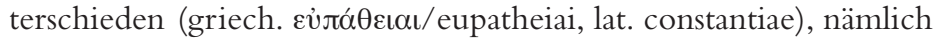
Wollen, Freude und Vorsicht. Das Wollen ist danach die ins Positive gewendete Form von Begierde, die Freude das positive Pendant zur Fröhlichkeit, die Vorsicht das positive Pendant zu Angst. Diese positiven Affekte (Wollen, Freude und Vorsicht) nahmen die Stoiker - dem Referat bei Cicero zufolge - auch für den Weisen an, also das Ideal des gelingenden Lebens. ${ }^{36}$ Für die Traurigkeit nahm die stoische Affektenlehre jedoch an, dass es hierfür kein positives Pendant gibt. ${ }^{37}$ Augustin stellt die Übereinstimmung seiner Affektenlehre mit der stoischen Affektenlehre heraus, wobei er allerdings deutlich macht, dass seiner Auffassung nach auch die Traurigkeit eine positive Seite haben kann. ${ }^{38}$ Dazu zitiert er 2. Kor 7,8-11, wo Paulus davon

35 Wichtige Parallelstellen sind De gratia novi testamenti 45-53 (CSEL 44, 193,3200,10 Goldbacher), vgl. dazu Pierre Descotes, Les deux craintes, in: Euvres de saint Augustin. 3e série. Premières réactions antipélagiennes II. La grâce de la nouvelle alliance/De gratia novi testamenti, texte établi, traduit et commenté, Bibliothèque Augustinienne vol. 20B, Paris 2016, 481-488; und Iohannis evangelii tractatus 41 (CCSL 36, 357,1-365,24 Willems), vgl. dazu Marie-François Berrouard, La dialectique de la crainte et de la joie chez le chrétien, in: Homélies sur l'Évangile de saint Jean. Homélies XXXIV-XLIII, in: CEuvres de saint Augustin. 9e série. Traités sur saint Jean, Bibliothèque Augustinienne 73A, Paris 1988, 492-497.

${ }^{36}$ Cicero, Tusculanae disputationes 4,6,11-13 (Bibliotheca Teubneriana M. Tullius Cicero Fasc. 44, 366,21-368,8 Pohlenz), gebraucht folgende Begriffe: perturbatio (= $\pi \dot{\alpha} \theta$ os): libido, laetitia, metus, aegritudo und für die positiven Affekte beim Wei-

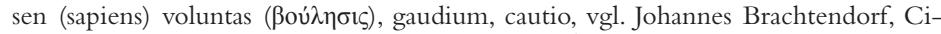
cero and Augustine on the Passions, in: Revue d'Études Augustiniennes 43 (1997), 289-308, hier 290-292; Adam Trettel, Desires in Paradise. An Interpretative Study of Augustine's City of God 14, Bd. 8, Paderborn 2019, 66-67.

37 Augustin, De ciuitate dei 14,8 (CCSL 48, 423,1-18 Dombart/Kalb).

${ }^{38} \mathrm{Vgl}$. Brachtendorf, Cicero and Augustine, 301-302. 
spricht, dass sein Brief die Adressaten vielleicht betrübt (contristare) hat, was ihn jedoch nicht reut, da ein gottgemäßes Betrübtsein zur Bußfertigkeit Gott gegenüber führt. ${ }^{39}$ Das führt Augustin zu seinem Ergebnis, dass er seine Beschäftigung mit der stoischen Affektenlehre aus dem neunten Buch von De cinitate dei bestätigt sieht. ${ }^{40}$ Für ihn steht fest, dass die Menschen alle vier Affekte erleben, positiv oder negativ. Diejenigen, die zur Gottesstadt gehören, haben sie als positive Affekte. Konkret bedeutet das: Sie fürchten die ewige Strafe, sie erwünschen das ewige Leben, sie sind traurig über die noch ausstehende Erlösung auch des Körpers, sie freuen sich aber in der Hoffnung, dass der Tod bereits besiegt ist. Sie haben Angst $\mathrm{zu}$ sündigen, sie wünschen sich, beharrlich in ihrem Glauben zu bleiben. Sie sind betrübt über die Sünden und freuen sich über gute Werke. ${ }^{41}$ Ein Sonderfall besteht im Hinblick auf die Versuchungen, denn der biblische Beter hat einerseits Angst davor, bittet andererseits Gott doch darum, versucht und geprüft zu werden, ist in den Versuchungen betrübt wie Petrus (Mt 26,75) und froh wie Jakobus, der zur Freude angesichts von Versuchungen aufruft (Jak 1,2). ${ }^{42}$

Für das Thema Angst ist die soziale Funktion der Angst wichtig. Die Christen haben die Affekte nicht nur um ihrer selbst willen, sondern auch um derer willen, von denen sie sich wünschen, dass sie befreit werden, und um die sie Angst haben, dass sie zugrundegehen. Paulus wird dafür zum Exempel par excellence. Er hat diese Affekte für die Adressaten seiner Briefe. Er freut sich mit den Fröhlichen und weint mit den Weinenden, er hat nach außen hin Kämpfe, nach innen hin Angstzustände (timores) und er streitet sich mit den Korinthern aus Angst, dass sie verführt werden. Er hat sogar Trauer und Herzschmerz (dolor cordis) um die, die ihre eigene Gerechtigkeit aufrichten wollen. ${ }^{43}$

Allerdings ändern auch diese positiven Affekte nichts daran, dass sie in dem gegenwärtigen Leben in einer gewissen Weise unbeherrschbar bleiben. Sie kommen über einen, auch wenn man es nicht will. So kommt es, dass man auch weint, obwohl man es nicht will. Das wird sich erst im eschatologischen Leben ändern. Das führt zu einer ambivalenten Haltung gegenüber dem Apathieideal. Auf der einen Seite kann das Apathieideal insofern geteilt werden, als

\footnotetext{
${ }^{39}$ Augustin, De ciuitate dei 14,8 (CCSL 48, 425,80-97 Dombart/Kalb).

40 Augustins Rückverweis bezieht sich auf De ciuitate dei 9,4-5 (CCSL 47, 251,1255,44 Dombart/Kalb).

${ }^{41}$ Augustin, De ciuitate dei 14,9 (CCSL 48, 426,4-12 Dombart/Kalb).

42 Augustin, De ciuitate dei 14,9 (CCSL 48, 426,21-32 Dombart/Kalb).

${ }^{43}$ Augustin, De ciuitate dei 14,9 (CCSL 48, 427,47-55 Dombart/Kalb).
} 
dass damit ausgedrückt wird, dass einen nicht länger Affekte oder Emotionen übermannen, die sich gegen die Vernunft des Menschen stellen (so dass er innerlich zerrissen ist und gerade nicht das tut, was er für richtig hält). Das wird aber im eigentlichen Sinne erst im Eschaton erreichbar sein. ${ }^{44}$ Auf der anderen Seite kann man aber das Apathieideal nicht in dem Sinn verstehen, dass - etwa im ewigen Leben - überhaupt keine Affekte und Emotionen mehr stattfinden. Man kann zwar sich gut vorstellen, dass es da nicht mehr die Angst und die Traurigkeit geben wird, weil sonst die Glückseligkeit beeinträchtigt wäre - aber ohne Liebe und Freude kann man sich die vollkommene Glückseligkeit eben auch nicht vorstellen. ${ }^{45}$

Genau besehen, muss man auch bei der Angst zwischen der Angst unterscheiden, die von der Liebe verdrängt wird (wie es ja auch 1. Joh 1,8 beschreibt: Die vollkommene Liebe vertreibt die Angst), und der Angst, mit der Paulus sich um die Korinther sorgt. Es gibt diese reine Angst (timor castus), womit Augustin wiederum auf Ps 18,10 rekurriert. Ps 18,10 ist dabei auch deswegen besonders passend, weil der Bibelvers davon spricht, dass diese reine Angst ewig bleibt (in saeculum saeculi). Diese Angst ist gerade nicht die Angst, die Böses zu vermeiden sucht, sondern eine Angst, die einen im Guten festhält (in bono tenens), und zwar - im Falle der eschatologischen Glückseligkeit - einem Guten, das nicht verloren werden kann. ${ }^{46}$ Diese reine Angst der eschatologischen Vollendung ist zugleich eine Abneigung gegen das Sündigen, aber nicht eine Angst, die dadurch entsteht, dass man um die eigene Schwäche weiß und das Sündigen als solches eine mögliche Bedrohung ist, sondern in dem Sinne, dass in einer Ruhe, die durch Liebe entsteht (tranquillitas caritatis) die Sünde nicht gewollt und vermieden wird (wobei das dabei benutzte Verb cavere mit dem lateinischen Substantiv cautio zusammenhängt, das bei den Stoikern die ins positiv gewendete Angst bezeichnete). ${ }^{47}$

Hat Augustin damit einerseits auf die eschatologische Erlösung der Menschen vorausgeschaut, fragt er sich anschließend, ob die Menschen vor dem Fall Affekte hatten oder nicht. Er fragt dies in besonderer Weise deswegen, weil Affekte die Glückseligkeit von Adam und Eva vor dem Fall beeinträchtigen könnten. Eigentlich glückselig kann man jemanden nicht nennen, der widerwillig an Trauer und Angst leidet. Es fragt sich auch, was eigentlich Anlass zu Trauer und Angst gegeben haben könnte. Im Paradies gab es ja

\footnotetext{
${ }_{44}$ Augustin, De ciuitate dei 14,9 (CCSL 48, 428,92-104 Dombart/Kalb).

${ }_{45}$ Augustin, De ciuitate dei 14,9 (CCSL 48, 428,104-114 Dombart/Kalb).

${ }_{46}$ Augustin, De ciuitate dei 14,9 (CCSL 48, 428,115-42,9,127 Dombart/Kalb).

${ }^{47}$ Augustin, De ciuitate dei 14,9 (CCSL 48, 429,127-132 Dombart/Kalb).
} 
weder den Tod noch irgendeine Krankheit, es gab alles, was der gute Wille haben wollte, und es gab nichts, was den Menschen körperlich oder seelisch beunruhigen könnte. Es gab Liebe zu Gott und der Menschen untereinander, damit auch Freude. ${ }^{48}$ Das bedeutet für Augustin auch, dass Adam und Eva in Ruhe lebten, also nicht bedroht von der Gefahr zu sündigen, oder davon, dass irgendein Übel geschehen würde. Man kann also gerade nicht annehmen, dass sie Angst hatten, dass sie sterben würden, wenn sie von der verbotenen Frucht essen würden, so dass es hier schon Begierde und Angst gegeben hätte. Denn eine solche Gesetzesbefolgung aus Angst würde selbst schon in sich sündhaft sein. Insofern muss man davon ausgehen, dass Adam und Eva wirklich glücklich waren, weder bedroht durch seelisch beunruhigende Affekte (perturbationes) noch durch körperliche Unannehmlichkeiten. ${ }^{49}$ Sie hatten gleichwohl Affekte, jedenfalls in dem positiven Sinne (also Liebe und Freude). Hierzu zählt Augustin auch die Sexualität, für die er nicht ausdrücklich sagt, dass Adam und Eva sie im Paradies von dem Fall schon gelebt haben, aber er sagt so viel, dass sie auch zu diesem Zustand der Glückseligkeit gehört hätte, in dem durch Fortpflanzung die Zahl der Menschen auf das vorherbestimmte $\mathrm{Maß}$ angewachsen wäre. ${ }^{50}$ Allerdings erweist sich diese Glückseligkeit insofern nicht als die höchst vorstellbare, weil sie nicht von der Gewissheit geprägt ist, dass der Mensch niemals sündigen und entsprechend sterben wird. Eine solche größere Glückseligkeit hätten die Menschen nur ohne Fall erreicht, dann hätten sie diese größere, höhere Glückseligkeit geschenkt bekommen, die auch die Engel bekommen haben, und die die Menschen jetzt eben nur nach der Auferstehung von den Toten erlangen können. ${ }^{51}$

Augustin klärt nicht, inwiefern Adam und Eva einen timor castus nach Ps 18,10 gehabt haben, und schließt timor (im engeren Sinne

\footnotetext{
${ }_{48}$ Augustin, De ciuitate dei 14,10 (CCSL 48, 430,1-15 Dombart/Kalb).

${ }^{49}$ Augustin, De ciuitate dei 14,10 (CCSL 48, 430,15-29 Dombart/Kalb).

${ }^{50}$ Augustin, De ciuitate dei 14,10 (CCSL 48, 430,29-431,34 Dombart/Kalb). Vgl. Trettel, Desires in Paradise, 96-98.

${ }^{51}$ Augustin, De ciuitate dei 14,10 (CCSL 48, 431,34-39 Dombart/Kalb). In Buch 11 hatte Augustin zwischen den Engeln unterschieden, die von Anfang an die Gewissheit erhalten haben, dass sie niemals fallen würden, und denen, die diese Gewissheit nicht erhalten haben. Letztere sind gleichsam naiv in ihrem gegenwärtigen Zustand ihrer guten Gottesbeziehung und ahnen nicht voraus (oder befürchten es), dass sie eines Tages fallen werden. In vergleichbarer Weise ist Adam vor dem Fall Augustin zufolge noch nicht von einer Ahnung einer mala voluntas betroffen, sondern erlebt sein Glück (ohne dass ihm die Frage des möglichen Falls bewusst würde), vgl. Augustin, De ciuitate dei 11,11-12 (CCSL 48,332,1-333,22 Dombart/Kalb).
} 
der Befürchtung von etwas Schlechtem) und dolor (als Erleben von Schlechtem) für das Paradies aus. ${ }^{52}$ Damit erweist sich Angst als ein Affekt, für den ein Übel als bedrohlich vorauszusetzen ist - dies setzt aber voraus, dass es tatsächlich schon eine mala voluntas gibt, dass also zumindest ein erstes Nachdenken über die Möglichkeit des Bösen entsteht. Erst dann, sobald die alternative Möglichkeit des Bösen in den Blick tritt, wird Angst möglich - selbst wenn sie sich auf andere und nicht auf einen selbst bezieht. Angst erweist sich so als Signatur der gefallenen Natur (und taucht als solche auch in Predigten als unruhestiftendes Potential auf), ${ }^{53}$ doch zugleich erweist sich an dem Beispiel Angst, dass diese gefallene Natur nicht einfach in den Urzustand zurückversetzt wird, sondern im Eschaton mehr erleben wird. Der erlöste Glaubende wird dann nicht mehr nur auf seine Angst und Unruhe zurückschauen, seine Angst etwa vor dem leiblichen Tod, seine Angst, in neue Sünden zu fallen oder gar vom Glauben ganz abzufallen, sondern er wird auch den von Liebe geprägten timor castus um andere, gerade auch um die, die nicht erlöst werden oder noch in Gefahr stehen, haben. ${ }^{54}$ Dies wird sich ihm mit einer Gewissheit verbinden, die diese neue Form von liebevoller Angst um andere mit der Gewissheit um die eigene Erlöstheit und das vollkommene Vertrauen auf Gottes Gerechtigkeit und Barmherzigkeit verbindet. Das kann der Mensch zwar jetzt schon im Gebet erhoffen (vgl. Ps. 100,1), es bleibt aber Gott überlassen, es dereinst umfassend zu realisieren.

- Prof. Dr. Volker Henning Drecoll ist Ordinarius für Alte Kirchengeschichte an der Evangelisch-Theologischen Fakultät der Eberhard Karls Universität Tübingen und Ephorus des Evangelischen Stifts Tübingen. Seine Forschungen beschäftigen sich insbesondere mit Basilius von Cäsarea und Gregor von Nyssa, dem Verhältnis von antikem Christentum und Philosophie (bes. Platonismus) und Augustin.

\footnotetext{
${ }^{52}$ Augustin, De ciuitate dei 14,9 (CCSL 48, 429, 143-145 Dombart/Kalb).

${ }^{53}$ Vgl. dazu Therese Fuhrer, Das "Zeitalter der Angst" als Konstrukt einer Rhetorik der Verunsicherung. Eine Analyse zweier Sequenzen aus Augustins Sermones ad populum, in: Irritationen. Rhetorische und poetische Verfahren der Verunsicherung, hg. v. Ramona Früh u.a., Berlin 2015, 61-85; Paul van Geest, Ante omnia igitur opus est Dei timore conuerti (doctr. chr. 2,7,9). Augustine's Evaluation of Fear, in:Tractatio scripturarum. Philological, exegetical, rhetorical and theological on Augustine's Sermons. Ministerium Sermonis II, ed. by Anthony Dupont/Gert Partoens/Mathijs Lamberigts, Bd. 65, Turnhout 2012, 441-463.

${ }^{54}$ Vgl. hierzu die Überlegungen von Trettel, Desires in Paradise, 84-85.
} 